Revista Temas Socio Jurídicos Vol. $38 \mathrm{~N}^{\circ} 76$ Enero - Junio de 2019

ISSN: 0120-8578

ISSN electrónico: 2590-8901

\title{
LA CIUDAD COMO ESPACIO DE LUCHA: MUJERES Y CONSTRUCCIÓN DEL ESPACIO URBANO
}

Almendra Aladro*

Recibido: Marzo 1 de 2019

Aprobado: Marzo 30 de 2019

\section{RESUMEN:}

Desde la década del 60 y a partir de la obra de Henri Lefebvre, la teoría comienza a redefinir la ciudad como derecho humano de carácter emergente, entendiéndola como el espacio de reproducción social natural del modo de producción capitalista. Algunos marcos normativos regionales como el brasilero y el ecuatoriano, han incorporado en sus textos fundamentales dispositivos protectorios de la ciudad como derecho, en miras a su acceso y goce en condiciones de igualdad. El artículo propuesto para el debate intenta constituirse como un aporte desde el enfoque de género para la construcción de resistencias urbanas al neoliberalismo.

Palabras clave: Derecho a la ciudad; feminismos; espacio urbano; políticas públicas; políticas locales.

Citar este trabajo como: Aladro, A. (2019). La ciudad como espacio de lucha: mujeres y construcción del espacio urbano. Temas Socio-Jurídicos, 38(76), pp. 11-23. https://doi.org/ 10.29375/01208578.3411

\footnotetext{
* Aspirante al título de Doctora en Derecho con mención en Ciencia Política (UNMDP), CONICET/CIDDH Alicia Moreau (CIC-UNMDP), Mar del Plata, Argentina. Orcid: https:// orcid.org/0000-0003-2658-6794. Correo Electrónico: aaladro@mdp.edu.ar
} 


\title{
THE CITY AS A SPACE FOR A FIGHT: WOMEN AND BUILDING URBAN SPACES
}

\begin{abstract}
:
Since the 1960s, and based on the works of Henri Lefebvre, the theory began to redefine the city as a human right of an emerging character, understanding it as a space for social natural reproduction of the means of capitalist production. Some regional regulatory frameworks, including the Brazilian and the Ecuadorian, have included in their texts fundamental protective devices of the city as a right, with a view to access and enjoyment thereof in equal terms. The presentation for this debate attempts to constitute itself as a contribution from the basis of gender for the construction of urban resistance to neoliberalism.
\end{abstract}

Keywords: Right to the city; feminism; urban space; public policies; local policies.

\section{A CIDADE COMO ESPAÇO DE LUTA: MULHERES E CONSTRUÇÃO DO ESPAÇO URBANO}

\section{RESUMO:}

Desde os anos 60 e a partir do trabalho de Henri Lefebvre, a teoria começa a redefinir a cidade como um direito humano de natureza emergente, entendendo-a como o espaço de reprodução social natural do modo de produção capitalista. Alguns marcos regulatórios regionais, como o brasileiro e o equatoriano, incorporaram os dispositivos de proteção da cidade como direito em seus textos fundamentais, com vistas ao seu acesso e desfrute em condições de igualdade. O artigo proposto para o debate tenta constituir-se como uma contribuição da perspectiva de gênero para a construção de resistências urbanas ao neoliberalismo.

Palavras-chave: Direito à cidade; feminismo; espaço urbano; política pública; políticas locais

\section{INTRODUCCIÓN}

En la actualidad, la Marea Violeta ha puesto en crisis el lugar social que ocupan las mujeres y se ha constituido como una suerte de renacimiento en la organización de su lucha en Argentina. El tema se ha instalado en las agendas pública y legislativa, y ha generado debates históricos, como los suscitados por el tratamiento de los proyectos de ley de interrupción voluntaria del embarazo y de educación sexual integral. 
La actual coyuntura interpela a la sociedad en su conjunto sobre el patriarcado como modo de opresión y sobre el machismo como práctica de la vida cotidiana. El escenario en el cual las luchas de las mujeres cis y transgénero se visibilizan y performan lo constituyen las ciudades, como otrora el centro de encuentro frente al avasallamiento de los trabajadores fue la fábrica, al iniciarse el proceso de industrialización descrito en la obra de Marx y Engels.

El protagonismo de los espacios urbanos en la lucha social fue el tema central de la investigación del sociólogo Henri Lefebvre, quien sostiene que "un proceso vinculado a la industrialización, pero diferente y específico, la urbanización, se iniciaba en tiempos de Marx", no obstante "no supo ni pudo descubrir en la producción de lo urbano la finalidad, el objetivo y el sentido de la producción industrial" (1968, p.235) ${ }^{1}$. En este sentido, el autor no concibe el surgimiento de las ciudades con la industrialización, puesto que incluso en su obra reconoce su preexistencia, sino que identifica que en el modo de producción capitalista es donde se agrupa la mano de obra asalariada, los mercados donde se introducen las mercancías y la dirección de los negocios.

A partir de esta base teórica, Lombardo sostiene que existe una articulación entre "la construcción del espacio, el proceso de producción y circulación del capital, la reproducción de la fuerza de trabajo y del capital, y las contradicciones derivadas de la base en que se asienta la apropiación del trabajo" (2012, p. 30). Sintetiza esta idea con claridad al tomar como referencia la obra de David Harvey (2006) citado en Lombardo (2012, p.31), explicando que "las contradicciones internas del capitalismo se expresan mediante la formación y modificación incesante de los paisajes geográficos".

El autor centra la observación del problema de la construcción de la ciudad en el concepto de reproducción social de la vida en un territorio, el cual entiende como:

Un proceso en el transcurso del cual los actores sociales de un territorio (...) realizan actividades, acciones y prácticas, crean herramientas y conforman relaciones y mecanismos de todo tipo articulados con sus necesidades, deseos y carencias individuales y colectivas, con el fin de construir las condiciones materiales y no materiales para el desarrollo de sus vidas en el lugar.

El resultado es un sistema, así como una construcción socio espacial, en que se generan mecanismos para funcionar, sostenerse, modificarse y transformarse con el fin de perpetuar las estructuras fundamentales del sistema en el tiempo. (Lombardo, 2012, p.37).

\footnotetext{
El destacado pertenece al autor.
} 
Entre otras fuentes, Lombardo cita a Carlos (2008) para la elaboración de este concepto. La autora parte de la afirmación de que "el espacio geográfico se constituye como condición, medio y producto de la reproducción de la sociedad en su totalidad, englobando varias escalas" (2008, s/n), para luego explicar que la reproducción del capital se relaciona con un proceso más complejo en el que se articulan todas las dimensiones de una formación social, y abogar por un análisis de la construcción del espacio "donde pueda pensarse al individuo no solo como fuerza de trabajo, permitiendo pensarlo, también, como sujeto de la producción del espacio" C arlos (2008) citada por Lombardo (2012, p.39).

Desde este entendimiento, la situación de las mujeres es particularmente compleja y los estudios sobre los vínculos entre patriarcado y capitalismo inscritos en la tradición marxista han omitido, en su mayoría, el impacto de las relaciones de subordinación basadas en la discriminación de género en la vida cotidiana, lo cual repercute en una visión incompleta respecto de los usos de los espacios urbanos por parte de las mismas. Esta consecuencia de la dicotomía público/privado, que tiene como base ideológica el liberalismo político lockeano y que es uno de los ejes centrales de la crítica feminista (Pateman, 2009), lleva a un entendimiento inacabado del fenómeno complejo que constituye el hacer ciudad, es decir, la participación de ciudadanas y ciudadanos en el gobierno de la misma. Despegarse de esta dicotomía para recuperar las ciudades:

(...) significa reconocer que las mujeres están situadas en la encrucijada entre producción y reproducción, entre la actividad económica y el cuidado y atención de los seres humanos y por lo tanto entre el crecimiento económico y el desarrollo humano. Ellas son las trabajadoras de ambas esferas: las más responsables y por lo tanto las que arriesgan más, las que sufren cuando las dos esferas funcionan de manera opuesta, y las más conscientes de la necesidad de una mejor integración entre las dos. (Sen, 1996, p. 60).

Desde estas bases teóricas, la presente ponencia se propone realizar un aporte al debate desde un enfoque de género para la construcción de resistencias urbanas al capitalismo en su etapa neoliberal. La exposición se dividirá en tres apartados en los que se desarrollarán las líneas de conocimiento más relevantes en materia de 1) el origen, evolución y recepción normativa del derecho a la ciudad; 2) las contribuciones destacadas desde la crítica feminista respecto del análisis del fenómeno de la construcción de las ciudades; y 3) la potencialidad de los Encuentros Nacionales de Mujeres como forma de resistencia urbana, tanto por la dinámica de su funcionamiento como por las peculiaridades de su emplazamiento territorial itinerante y federal.

Posteriormente se identificarán los desafíos y perspectivas en la lucha de las mujeres por la recuperación de las ciudades. 


\section{EL DERECHO A LA CIUDAD}

\section{Concepto y desafíos}

El hito más trascendente en la elaboración del concepto de derecho a la ciudad es el libro de Henri Lefebvre que, precisamente, se titula El derecho a la ciudad. Allí, Lefebvre inicia su estudio sobre la construcción de la ciudad y afirma:

La ciudad, y por consiguiente lo urbano, es el resultado de una historia, de una praxis social, de personas y grupos determinados que realizan esta obra en condiciones históricas. De esto surge la ciudad como obra, como resultado de esta praxis, así la vida cotidiana se torna obra, apropiación del espacio, uso. (1969, p.68)

Esta idea central es alcanzada a través de un análisis desde el materialismo dialéctico del fenómeno de las ciudades y de la urbanización que se desarrolló tras la explosión de la industrialización, pero no ya como meros asentamientos, sino como espacio donde se desarrolla la lucha de clases, por lo que su forma y construcción responde a la lógica del capital. Así las ciudades se fragmentan y se reconstruyen en función de la organización de la producción, perdiéndose el valor de uso del espacio urbano frente a la hegemonía de su valor de cambio.

Por su parte, Harvey (2012) sostiene que el derecho a la ciudad es un derecho colectivo de todas las personas que habitan, acceden y usan la ciudad, y supone no solamente el derecho a usar aquello que ya existe, sino también a definir y crear lo que debería existir en los espacios urbanos, con miras a la satisfacción de la necesidad de llevar una vida digna y plena en dicho entorno.

Como perspectiva superadora, Edward Soja incorpora a la idea de ciudad como espacio de la lucha de clases propuesta por Lefebvre y su recuperación a través de prácticas insurgentes que vehiculicen la participación de la ciudadanía en la construcción de los espacios urbanos esbozada por Harvey con la noción de justicia espacial. Desde este concepto, Soja critica las posturas marxistas tradicionales respecto a la ciudad, por excluir a través del concepto de lucha de clases perspectivas como el género, las migraciones, las cuestiones étnicas y culturales, que son aristas clave al momento del análisis de la construcción y prácticas urbanas. Metodológicamente, el autor propone la "trialéctica" o estudio del tercer espacio, una forma de abordaje de la ciudad como objeto de estudio que contempla tres dimensiones de análisis, a saber: 1) el mundo real y material; 2) las representaciones sociales y 3 ) el espacio producido a partir de las dimensiones 1 y 2 (Soja, 2014).

Jordi Borja, geógrafo urbanista catalán, se pregunta: —¿Qué es una ciudad? y se responde: 
-Un lugar con mucha gente. Un espacio público, abierto y protegido. La ciudad construida urbanismo en América Latina y protegido. Un lugar, es decir, un hecho material productor de sentido. Una concentración de puntas de encuentros. En la ciudad, primero son las calles y plazas, los espacios colectivos, luego vendrán los edificios y las vías. El espacio público define la calidad de la ciudad, porque indica la calidad de vida de la gente y la cualidad de la ciudadanía de sus habitantes. (Borja, 2001,p.391)

Pero frente a esa ciudad del deseo, Borja explica el fenómeno de la ciudad fragmentada:

(...)la deformación del urbanismo funcionalista combinando zooning y privatización, caricatura perversa del movimiento moderno, crea una nueva imagen de la 'ciudad emergente' en la que las piezas, la arquitectura de los objetos-mercancía, substituye la ciudad del intercambio y de la diversidad. La ciudad fragmentada es una ciudad físicamente segregada, socialmente injusta, económicamente despilfarradora, culturalmente miserable y políticamente ingobernable. (Borja, 2001, p.392)

La actualidad de las ciudades lleva a plantear desde estas perspectivas la lucha por la ciudad, su reivindicación como derecho y no como privilegio de clase. Borja afirma:

Construir hoy la ciudad del siglo XXI es tener un proyecto de ciudadanía, ampliar los derechos de tercera generación, el derecho al lugar y a la movilidad, a la ciudad refugio y a la identidad local, al autogobierno y a la diferencia, a la igualdad jurídica de todos los residentes y a la proyección exterior. (Borja, 2001, p.395)

La lectura conjunta de estos autores permite observar con claridad la evolución conceptual de la ciudad y de las estrategias metodológicas planteadas tanto para su abordaje como objeto de estudio, como las diagramadas para la recuperación de los espacios urbanos.

\section{Recepción normativa}

El derecho a la ciudad se ha caracterizado como humano y emergente (Guillén Lanzarote, 2011) y su desarrollo normativo es posterior al reconocimiento del derecho humano a la vivienda (Pisarello, 2011).Correa Montoya se refiere a su contenido como derecho, reafirmando su complejidad y estableciendo cuatro ejes fundamentales para su consagración:

1) como posibilidad social, es decir, "como una forma o modelo concreto en que la ciudad, lugar, objeto e institución privilegiada, se ofrece y posibilita a sus ciudadanos"; 2) como goce de derechos en los contextos urbanos, o sea, atado "a ese modelo basado en derechos (...) que exige que todos los ciudadanos se beneficien de lo que las ciudades tienen para ofrecer"; 3) como derecho complejo, "la posibilidad de construir 
espacios de encuentro, deliberación e incidencia en los destinos colectivos (...) de construir y hacer ciudad, de vivirla, disfrutarla e incidir en el destino de una ciudad digna que permita la distribución de recursos"; 4) como derecho colectivo, implicando "ampliar el enfoque tradicional orientado a mejorar la calidad de vida de las personas desde la vivienda y el vecindario, para hacerlo trascender a una escala más amplia, la de la ciudad y sus alrededores rurales". (Correa Montoya, 2010, p.40-44)

Desde el punto de vista estrictamente jurídico, existen numerosos instrumentos internacionales que lo han dotado de contenido, tales como la Declaración Universal de los Derechos Humanos, el Pacto de Derechos Económicos, Sociales y Culturales, la Resolución 64/292 de la Asamblea de las Naciones Unidas , la Carta Europea de Salvaguarda de los Derechos Humanos en la Ciudad, el Estatuto de las Ciudades (Brasil, 2001), siendo este último ratificado en el Foro Social Urbano de las Américas (Quito, 2004), el Foro Mundial Urbano (Barcelona, 2004) y en el Foro Social Mundial (Porto Alegre, 2005), la Carta Mundial por El Derecho a la Ciudad y la Declaración Universal de los Derechos Humanos Emergentes.

Dada la complejidad de la ciudad como derecho, según el Comité Promotor de la Carta de la Ciudad de México por el Derecho a la Ciudad, otras herramientas jurídicas a contemplar para su acceso y goce que deben tenerse en cuenta son:

- Declaración Universal de los Derechos Humanos.

- Declaración Americana de los Derechos y Deberes del Hombre.

- Pacto Internacional de los Derechos Civiles y Políticos.

- Convención Americana sobre Derechos Humanos (Pacto de San José).

- Protocolo Adicional a la Convención Americana sobre Derechos Humanos en materia de Derechos Económicos, Sociales y Culturales (Protocolo de San Salvador).

- Convención Internacional sobre la Eliminación de Todas las Formas de Discriminación Racial.

- Convención sobre la Eliminación de Todas las Formas de Discriminación contra la Mujer.

- Convención contra la Tortura y Otros Tratos o Penas Crueles, Inhumanos o Degradantes.

- Convención sobre los Derechos del Niño y sus dos protocolos facultativos, uno relativo a la venta de niños, la prostitución infantil y la pornografía infantil, y el otro relativo a la participación de niños en los conflictos armados. 
- Convención Internacional sobre la Protección de los Derechos de Todos los Trabajadores Migratorios y de sus Familiares.

- Convención Internacional para la Protección de Todas las Personas contra las Desapariciones Forzadas.

- Convención sobre los Derechos de las Personas con Discapacidad.

- Convención para Prevenir, Sancionar y Erradicar la Violencia contra la Mujer (Convención de Belém do Pará) (2010, p. 9-10).

En la región, existen hitos normativos sobre la materia, originados principalmente desde la lucha social por el derecho a la ciudad. En Brasil encontramos la Constitución Federal de Brasil de 1988 (art. 182, 183), la cual contó con el aporte de más de 100.000 organizaciones sociales y de adhesiones individuales de participantes del Movimiento Nacional de Reforma Urbana, que formularon la Enmienda Popular de Reforma Urbana (Fernandes, 2010) y el Estatuto del Derecho a la Ciudad del año 2001 (Ley Federal 10257), texto que "pone sobre el tapete la cuestión urbana y la inserta en la agenda política nacional, en un país marcado hasta fechas recientes por la cultura rural" (Maricato, 2010, p.5).

A nivel nacional, Argentina no cuenta con un dispositivo federal que garantice el acceso y goce de la ciudad como derecho, aunque existen dispositivos que reconocen los derechos humanos que quedan bajo su marco (Const., 1994, art. 20, 25, 37, 41, 43, 75 inc.22). A nivel local, se destaca la Ley de Acceso Justo al Hábitat de la Provincia de Buenos Aires, la cual tiene como objetivo general "la promoción del derecho a la vivienda y a un hábitat digno y sustentable" y como objetivos específicos a) Promover la generación y facilitar la gestión de proyectos habitacionales, de urbanizaciones sociales y de procesos de regularización de asentamientos urbanos informales; b) Abordar y atender integralmente la diversidad y complejidad de la demanda urbano-habitacional; y c) Generar nuevos recursos en miras a la reducción de la especulación sobre el valor de cambio del suelo (Ley 14449, art. 1). Así mismo, reconoce el derecho a la ciudad como uno de los principios rectores de las políticas de vivienda y hábitat de la provincia (art. 10), y le da contenido al mismo a través de la conceptualización del acceso y goce a la ciudad y la vivienda, entendiendo a dichos elementos como: a) un lugar adecuado para vivir en condiciones que favorezcan la integración plena a la vida urbana; b) acceder a los equipamientos sociales, a las infraestructuras y a los servicios; c) desenvolver apropiadamente las actividades sociales y económicas; y d) usufructuar de un hábitat culturalmente rico y diversificado (art. 11).

\section{APORTES DESDE LA PERSPECTIVA DE GÉNERO PARA LA REAPROPIACIÓN DE LAS CIUDADES}

En la última década, el estudio del derecho a la ciudad ha evolucionado incorporando la perspectiva de género al análisis de las políticas, con particular 
énfasis en la desigualdad del acceso a la ciudad, como consecuencia de una discriminación basada en el género. Explica Falú que "las mujeres permanecerán invisibles en las ciudades, relegadas al ámbito privado del hogar, lo que tendrá sustento en la fuerte división del trabajo sexual imperante" (2016, p.160). En esta afirmación se evidencia la presencia de la dicotomía público/privado como eje estructurante de las desigualdades urbanas basadas en el género. Las ciudades, espacio de toma de decisiones (vida pública), se constituyen como un espacio propiamente del varón, mientras que el hogar es el dispositivo en el cual se confina a la mujer (vida privada) pero donde el hombre también conserva la esfera de toma de decisiones. Sobre esta díada Carole Pateman realiza una interesante observación en su crítica al liberalismo, afirmando que:

(...) la crítica feminista contemporánea a la dicotomía entre lo público y lo privado se basa en la misma perspectiva lockeana de las dos categorías; al igual que (en esta interpretación de) la teoría lockeana, las feministas consideran que la vida doméstica es privada por definición. Sin embargo, rechazan el supuesto en virtud del cual la separación entre lo privado y lo público se sigue inevitablemente de las características naturales de los sexos y sostienen, por el contrario, que solo resulta posible una correcta comprensión de la vida social liberal cuando se acepta que las dos esferas -la doméstica (privada) y la sociedad civil (pública) - presuntamente separadas y opuestas están inextricablemente interrelacionadas. Dicho de otra forma, que son las dos caras de la misma moneda, el patriarcalismo liberal. (Pateman, 2009, p.42-43)

Y desde esta lógica, identifica que:

(...) el liberalismo conceptualiza la sociedad civil prescindiendo de la vida doméstica, esta última sigue siendo «olvidada» en las discusiones teóricas. Así pues, la separación entre privado y público se vuelve a establecer como una división dentro de la propia sociedad civil, dentro del mundo de los varones. Por tanto, la separación se expresa de diferentes maneras, no solo en términos de privado y público sino también, por ejemplo, en términos de "sociedad" y «Estado", de «economía» y "política», de «libertad" y "coerción» o de "social» y "político». Además, en esta versión de la separación de lo privado y lo público, una categoría, lo privado, empieza a vestir pantalones-. (Pateman, 2009, p.43).

Al aplicar las reflexiones de Pateman, hay una conclusión obvia pa ra este trabajo: la planificación de las ciudades "evidencia problemas de orden ontológico y epistemológico, al reproducir desigualdades y subordinación en sus políticas" (Falú, 2016, p.160). Si la ciudad es el espacio donde se desarrolla la reproducción social de la vida (Lombardo, 2012), la exclusión de las mujeres en su planificación es una estrategia de replicación de las prácticas machistas y refleja graves consecuencias derivadas de la discriminación urbana basada en el género. 
Desde esta toma de posición, Shelly Buckingham (2010) describe cinco problemas en el acceso y goce del derecho a la ciudad por parte de las mujeres; los cuales, a su criterio, deben constituirse como objetivos de políticas simultáneas para lograr un cambio real en la situación de este colectivo social: 1) la falta de seguridad en ambientes urbanos; 2 ) la deficiencia en la infraestructura y transporte públicos; 3) la falta de proximidad entre viviendas, servicios y empleos; 4) la persistencia de la dicotomía de esferas públicas y privadas; y 5) el déficit de participación en la toma de decisiones, gobernanza y planificación de las ciudades.

Con respecto a la relevancia de este nuevo abordaje, Fernando Manero Miguel (2010) destaca que la toma de conciencia social sobre las formas de desigualdad que afectan a las mujeres, conduce a que la acción pública tienda a asumir como imperativa la nectesidad de incorporar esta cuestión dentro de sus preocupaciones estratégicas como guía en la toma de decisiones con intencionalidad correctora.

\section{REFLEXIONES FINALES: PRÁCTICAS URBANAS REBEL- DES DE LAS MUJERES}

En este trabajo se ha evitado la elaboración de las clásicas reflexiones o conclusiones finales, porque lejos de ser un tema cerrado, el debate sobre la planificación urbana y los aportes de las mujeres a la misma se encuentra en el candelero. En oposición a dicha forma de escritura, se trae a debate el Encuentro Nacional de Mujeres (ENM), un evento autoconvocado y autogestionado que se desarrolla de forma anual y de manera ininterrumpida desde hace 33 años en Argentina. En él se congregan masivamente mujeres con ideas políticas disímiles con el objetivo de problematizar los ejes centrales de la agenda feminista nacional.

En el último ENM, realizado en la ciudad de Resistencia (provincia de Chaco), se estima que participaron alrededor de 75.000 mujeres, quienes durante tres días enteros ocuparon y resignificaron los espacios urbanos locales por trigésima segunda vez.

Los talleres de debate se realizan en las escuelas y universidades. Centros deportivos y educativos prestan sus lugares para el pernocte, aseo y alimentación. Las plazas se llenan de ferias y manifestaciones artísticas, y en las calles la marcha de cierre desborda de cuerpos que se enfrentan al patriarcado que los oprime y reprime.

Más allá de los temas abordados durante los ENM (Aladro, 2017), estos representan una práctica urbana insurgente porque "introducen en la ciudad nuevas identidades y prácticas que disturban las historias establecidas" (Holston, 1995, p.48). Las ciudades elegidas por las mujeres al finalizar el evento y negociadas con los poderes locales por parte de las comisiones organizadoras a cargo, son forzadas a transformarse por tres 
días, a permitir a las mujeres caminar por las calles en libertad, disponer de sus espacios, vivir sus corporalidades sin miedos, delegar el cuidado familiar y las tareas domésticas. Durante los encuentros, las mujeres irrumpen en la ciudad y derriban el muro divisorio entre los espacios público y privado.

Entendiendo que el concepto de práctica insurgente elaborado por Harvey (2012) invoca una idea de permanencia inherente a la gestión urbana, en este escrito se postula la idea de práctica rebelde como puntapié inicial para la constitución de expresiones de la trascendencia que esboza el autor. Por los motivos que se explicaron en los párrafos anteriores, este trabajo defiende la idea de que los ENM como eventos de arraigo territorial itinerante, pero de indudable significado social, constituyen una práctica rebelde frente al patriarcado y al modo de producción capitalista en sus actuales estadios de desarrollo.

Finalmente, se deja planteada la idea de que la actual Marea Violeta en sus expresiones públicas, también constituye una práctica rebelde de masas cuya evolución debe seguirse de cerca en el estudio del derecho a la ciudad desde el punto de vista de las mujeres.

\section{REFERENCIAS}

Aladro, A. (2017). El Encuentro Nacional de Mujeres como espacio de construcción del Derecho a la Ciudad desde la perspectiva de género. Slavin, P. y García, L. (Comp), Adelantos de Investigación en Ciencias Jurídicas y Sociales. Argentina, Mar del Plata: EUDEM.

Borja, J. (2001). La ciudad del deseo. En: Carrión, F. (Ed.). La ciudad construida urbanismo en América Latina (pp. 391-396). Quito, Ecuador: FLACSO.

Buckingham, S. (2010). Análisis del derecho a la ciudad desde una perspectiva de género. En: Sugranyes, A. y Mathivet, C. (eds.). Ciudades para tod@s. Por el derecho a la ciudad, propuestas y experiencias (pp. 5964). Santiago, Chile: Coalición Internacional para el Hábitat.

Carlos, A.F. (2008). De la "geografía de la acumulación" a la "geografía de la reproducción": un diálogo con Harvey. Scripta Nova Revista Electrónica de Geografía y Ciencias Sociales, XII (270), 143, s/n.

Comité Promotor de la Carta de la Ciudad de México por el Derecho a la Ciudad (2010). Carta de la Ciudad de México por el Derecho a la Ciudad. Recuperado de: https://www.equipopueblo.org.mx/descargas/Carta\%20de\%20la\%20Ciudad\%20de\%20Mexico\%20por\%20 el\%20Derecho\%20a\%20la\%20Ciudad.pdf [Fecha de consulta: 05 de octubre de 2018].

Correa Montoya, L. (2010). Algunas reflexiones y posibilidades del Derecho a la Ciudad en Colombia: Los retos de la igualdad, la partici- 
pación y el goce de los derechos humanos en los contextos urbano. Revista Jurídica de la Universidad de Palermo (Online) [Fecha de consulta: 05 de octubre de 2018].

Falú, A. (2016). La omisión de género en el pensamiento de las ciudades. En: Borja, J., Carrión, F. y Corti, M. (Ed.). Ciudades para cambiar la vida: Una respuesta a Hábitat III. Buenos Aires, Argentina: Editorial Café de las Ciudades.

Fernandes, E. (2010). El Estatuto de la Ciudad y el orden jurídico-urbanístico. En: Celso Santos Carvalho, Anaclaudia Rossbach (Org.). El Estatuto de la Ciudad: un comentario. Sao Paulo, Brasil: Alianza de las Ciudades.

Guillén Lanzarote, A. (2011). El derecho a la ciudad, un derecho humano emergente. En: Serie Derechos Humanos Emergentes 7: El derecho a la ciudad. Barcelona, España:Instituto de Derechos Humanos de Catalunya.

Harvey, D. (2006). La geografía de la acumulación capitalista: una reconstrucción de la teoría marxista.

Harvey, D. (2012). Ciudades rebeldes: Del derecho de la ciudad a la revolución urbana, Madrid, España: Ed. Akal.

Holston, J. (1995). Spaces of insurgent citizenship, en Planning Theory (Online) pp. 35-51 [Fecha de consulta: 05 de octubre de 2018].

Lefebvre, H. (1968). La vida cotidiana en el mundo moderno, Madrid, España: Alianza Editorial.

Lombardo, J. D (2012). La construcción del espacio urbano: Sus características en el Area Metropolitana de Buenos Aires. Los Polvorines, Argentina: Ediciones CICCUS.

Manero Miguel, F. (2010). Mujer y espacio urbano: Dimensión y operatividad de la perspectiva de género de las políticas públicas locales en el contexto de la Unión Europea, Revista Ciudades número 88, p. 1-20.

Maricato, E. (2010). El estatuto de la ciudad periférica. En: Santos Carvalho,C. Rossbach, A. (Org.). El Estatuto de la Ciudad: un comentario. Sao Paulo, Brasil: Alianza de las Ciudades.

Pateman, C. (2009). Críticas feministas a la dicotomía público/privado. En: Ávila Santamaría, R., Salgado, J. y Valladares, L. (Comp). El género en el derecho. Ensayos críticos. Quito, Ecuador: Ministerio de Justicia y Derechos Humanos.

Pisarello, G (2011). Del derecho a la vivienda al derecho a la ciudad: avatares de una historia.Serie Derechos Humanos Emergentes 7: El derecho a la ciudad. Barcelona, España: Instituto de Derechos Humanos de Catalunya. 
Sen, Gita. (1996). Una economía alternativa desde una perspectiva de género. Nuevos enfoques económicos: contribuciones al debate sobre género y economía (pp. 53-63). San José, Costa Rica.

Soja, E. (2014). En busca de la justicia espacial. Valencia, España: Tirant Humanidades. 